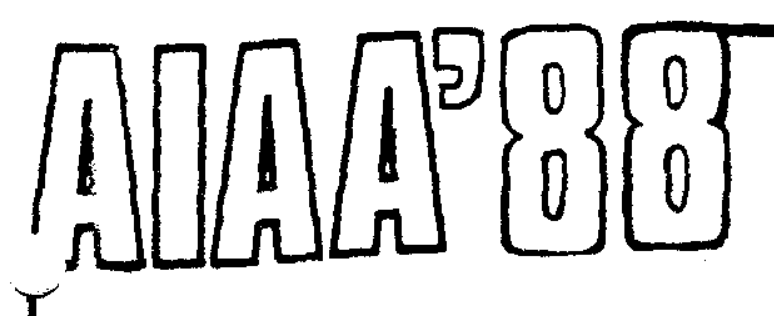

\title{
AIAA-88-0146 \\ Visualization of the Structure of Premixed Turbulent Flames
}

D. Feikema, W. Roberts, R. H. Chen, and J. F. Driscoll, Department of Aerospace Engineering, University of Michigan, Ann Arbor, Michigan

\section{AIAA 26th Aerospace Sciences Meeting January 11-14, 1988/Reno, Nevada}




\author{
Visualization of the Structure of Premixed Turbulent Flames \\ Douglas Feikema, W. Roberts, R. H. Chen and J. F. Driscoll \\ Department of Aerospace Engineering \\ University of Michigan \\ Ann Arbor, MI 48109-2140
}

\begin{abstract}
The present paper presents some preliminary results showing how the structure of a premixed flame changes as the level of turbulence and the integral length scale in the incident flow are varied. This study is a continuation of the work of Gulati and Driscoll, in which an atmospheric, relatively low Reynolds number turbulent flame was studied using laser velocimetry and Rayleigh scattering. Schlieren photographs show that cusp-shaped wrinkles occur, as has been documented in previous studies. In the present study it is found that the cusped wrinkles occur for both lean methane-air flames and lean propane-air flames, indicating that the wrinkles are not due to a thermo-diffusive instability in this case. The Reynolds number is increased by a factor of four by increasing the turbulence-producing grid spacing, and therefore increasing the integral size of the turbulence. However, such changes cause a decrease in the size of flame wrinkles. The wrinkles are not produced locally by eddies impinging on the flame, but are formed near the flame stabilization location and propagate up along the flame sheet.
\end{abstract}

\section{Introduction}

Previously, a study by Gulati and Driscoll ${ }^{1,2,3}$ showed how certain important velocity-density correlations vary in a pre- mixed flame. Laser velocimetry and laser Rayleigh scattering ${ }^{4}$ were used simultaneously to map out the velocity and scalar (density) fields. For those measurements, the following quantities were deduced: four of the six terms in the turbulent kinetic energy equation, the local mean reaction rate, the flux of turbulent kinetic energy, the "true" turbulence level, and the "apparent" turbulence associated with flamelet motion.

However, several additional measurements are useful to better understand the previous results of Gulati and Driscoll, and these measurements are discussed below. For example, since name speed was unambiguously determined for a range of conditions using a complete LDV velocity profile, it was decided to measure the integral length scale, to allow for comparison of the present flame speed with those of other researchers.

A schematic of a turbulent premixed flame is shown in Fig. 1. A fundamental question that has yet to be answered relates to how the incident turbulence wrinkles the flame sheet, thereby increasing the flame area, the reaction volume, and thus the reaction rate and burning velocity. Damkohler ${ }^{5}$ showed that the ratio of turbulent to laminar buming velocities $\left(S_{T} S_{L}\right)$ for a wrinkled flame equals the ratio of the actual flame sheet area $\left(A_{T}\right)$ to the crosssectional area $\left(A_{0}\right)$ shown in Fig. 1. By continuity $\rho S_{T} A_{0}$ equals $A_{T} \delta R R$ where $\delta$ is the flamelet thickness and $R R$ is the reaction rate. For a laminar flame $\rho S_{L} A_{0}$ equals $A_{0} \delta$ $R R$. By taking the ratio of the first equation to the second $S_{T} / S_{L}=A_{T} / A_{a}$. Therefore, any fundamental understanding of turbulent reaction rates requires an understanding of how much the flame surface is increased by wrinkling due to eddies. 
Ballar ${ }^{4}$ and Bradley ${ }^{7}$ have measured and analyzed values of buming velocity $\left(S_{T} / S_{L}\right)$ in the wrinkled flame regime as well as in the higher Reynolds number thick flame zone regime. In both works, the importance of turbulence length scales was emphasized, which was an improvement over Damkohler's theory. Ballal postutated how integral scale can effect flame speed. As modern flow visualization diagnostics become available, it should be possible to better quantify and verify the mechanisms by which turbulence increases the flame sheet area. Such visualization information also should prove useful in the development of discrete vortex models. Ashurst ${ }^{6}$ has shown how turbulence produces cusped-shaped wrinkles in a discrete vortex simulation of a premixed flame. More quantitative measurements could prove useful to further the development of such models. The present work is a first step in an effort to provide such measurements. It is noted that the present paper describes Schlieren photographs only; more advanced flow and flame visualization methods are in the process of being developed for this experiment and will be reported in the future.

\section{Experiment}

Premixed methane-air and propane-air flames were stabilized on one edge of the rectangular burner shown in Fig. 2. The reactants are passed through a turbulenceproducing grid whose mesh size was varied. The burner exit is $3.8 \mathrm{~cm} \times 2.54 \mathrm{~cm}$ and is surrounded on all sides by sheath air to prevent a shear layer from forming between the primary flow and the stagnant surroundings. Additional details about the burner are given in Refs. 1.3.

The flame is shown in Fig. 3. It appears to be nearly planar, having a brush thickness of one $\mathrm{cm}$, and is oriented at about 35 degrees from the flow direction. Attempts to obtain larger orientation angles (i.e. up to 90 degrees) resulted in flashback. The mean properties of such a flame are as onedimensional as physically possible. The flame orientation angle is large enough to keep the flame out of the wake of the stabilizing rim: thus the entire flame remains out of any wakes or shear layers and all turbulence is due only to the grid.
The fuel and air flows were monitored using calibrated rotameters and micrometer valves. A Schlieren system was used for both still photographs as well as movies at 4000 frames per second. A Xenon lamp was powered with a power source that allowed for continuous operation for alignment and movies, and for pulsed operation for still photographs. The Schlieren system employed 48-inch focal length mirtors and a horizontal knife edge.

The turbulence length scales were measured in the cold flow using a TSI Model $1050 \mathrm{Hot}$ Wire Anemometer and a Model 1052 Signal Linearizer. The system was operated in the constant temperature mode with an overheat ratio of 1.2. Power spectra were recorded on a Hewlett Packard analog spectrum analyzer.

\section{Results}

Turbulence levels within the flame brush were described in a previous paper ${ }^{1}$ and will not be repeated herein. The profiles of turbulent velocity fluctuations (such as shown in Fig. 4), gas density fluctuations. and velocity-density correlations were measured. A photograph of the wrinkles in the flame is seen in Fig. 5. The cusp-shaped wrinkles are similar to those reported in rod-stabilized flames. The cusps (i.e., pointed edges) point toward the products. Several possible mechanisms can cause the cusps: a thermodiffusive instability, ${ }^{5,9}$, a Taylor-Markstein instability, ${ }^{9}$ or perhaps some other mechanism. The wrinkles are shown in Fig. 6 to form near the flame stabilization point and move along the flame. Therefore, models which assume that turbulent eddies locally cause wrinkles as the eddy passes through the flame may be insufficient.

The cusped wrinkles observed are not thermo-diffusive in nature, as evidence by Fig. 7. A lean propane-air mixture cannot support a thermo-diffusive instability, ${ }^{5,9}$ yet a lean methane-air mixture will support such an instability. Yet the flames for both mixtures (Figs. 5 and 7) show similar types of wrinkles. The thermo-diffusive instability mechanism occurs when fuel-lean conditions occur and the fuel diffuses faster than air; thus a small wrinkle will be amplified as the 
reactants entering the cusped region become leaner due to diffusion.

The effects of increasing grid size and thus Reynolds number is shown in Figs. 8-10. The measured ratio of $S_{\top} / S_{L}$ increases from 3.5 to 4.9 , as expected, since the incident turbulence level increases. However, even though the grid becomes coarser and the scale of turbulence increases, the wrinkle size is observed to decrease. The wrinkles remain cusped even as the Reynolds number increases by a factor of four and the flame becomes even more turbulent. For some cases, the wrinkles are ten times greater than the integral scale of the turbulence, which was measured as described below. Damkohler's theory assumes that wrinkle size exactly equals the integral scale of incident eddies.

The decay of turbulence behind one grid is shown in Fig. 11. The decay is similar to that predicted by Batchelor. ${ }^{10}$ Integral length scales were measured from the hot wire power spectra, an example of which is shown in Fig.

12. Integral scale is $(\bar{U} / 4) E_{o} / \int_{0}^{\infty} E d f$ where $E_{0}$ is the zero frequency intercept of the spectrum. ${ }^{10}$

\section{Beferences}

1. "Measurement of Various Terms in the Turbulent Kinetic Energy Balance Within a Flame," Driscoll, J. F. and Gulati, A., Comb. and Elame, in press.

2. "Flame Generated Turbulence," Gulati, A. and Driscoll, J. F., Twenty-first Symp. (Intl.) on Combustion, 1988).

3. "Velocity-Density Correlations in a Premixed Flame," Gulati, $A$. and Driscoll, J. F., Comb.Sci Tech. 48, p $285,1986$.

4. "Mass Fluxes Measured in a Turbulent Nonpremixed Flame," Driscoll, J. F., Schefer, R. W. and Dibble, R. W., Nineteenth Symp. (Intl.) on Combustion, p 477, 1982.

5. Combustion. Flame and Explosions of Gases. Lewis, B. and Von Elbe, G., Academic Press, 1961.
6. Ballal, D., Proc. Royal Soc. London, A367: 485, 1979.

7. Andrews, G. E., Bradley, D. and Lwakabamba, S., Comb. and Elame 24, $285,1975$.

8. Ashurst, W., Comb.Sci.Tesh. 52, 325, 1987.

9. Combustion Fundamentals, Strehlow, R., McGraw Hill, 1985.

10. Theory of Hemogeneous Turbulence, Batchelor, G. K., Cambridge U. Press.

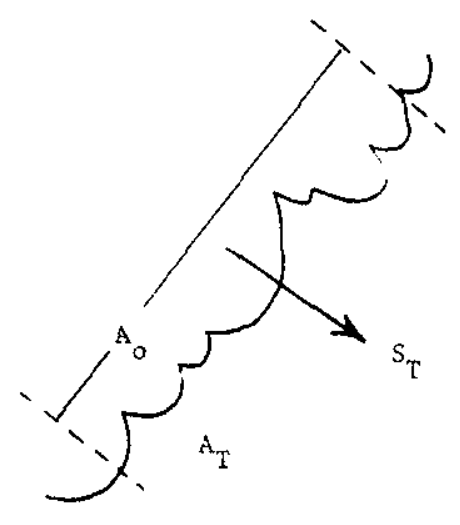

Figure 1, Schematic of a Premlxed Flame

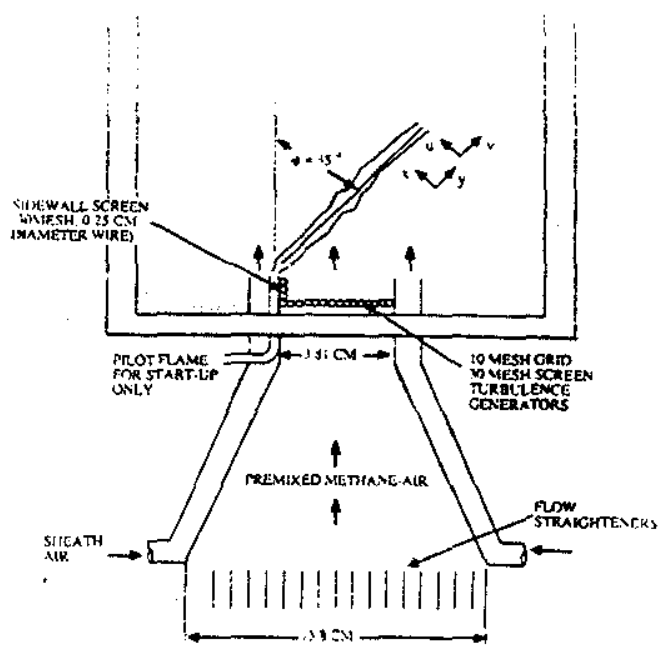

Figure 2. Schematic of the Burner 


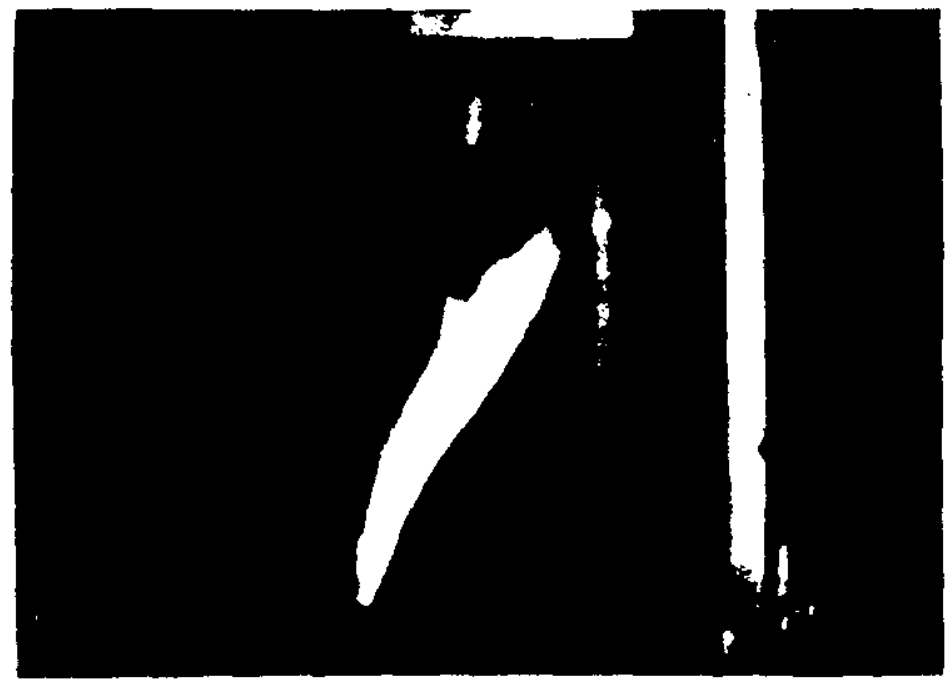

Figure 3. Photograph of the Turbulent Flame

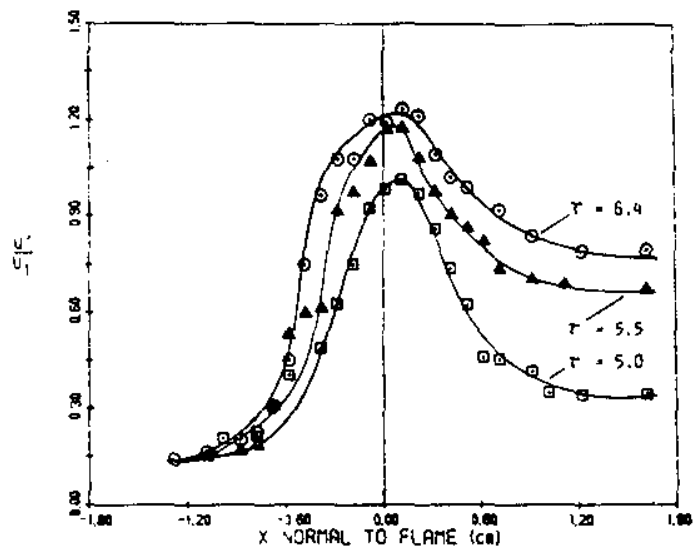

Figure 4. Profiles of velocity Fluctuations Within the Flame Using LDV

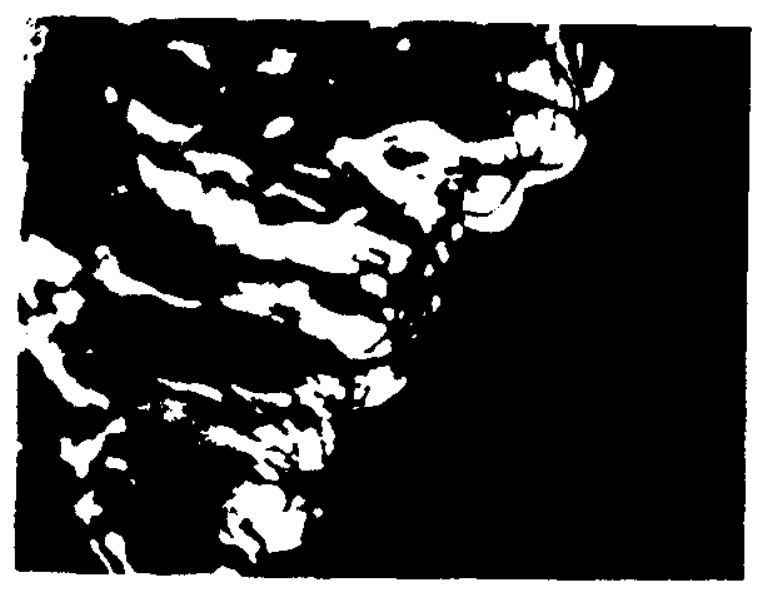

Figure 5. Schlieren Photograph of a MethaneAir Flame for $\emptyset=0.8$

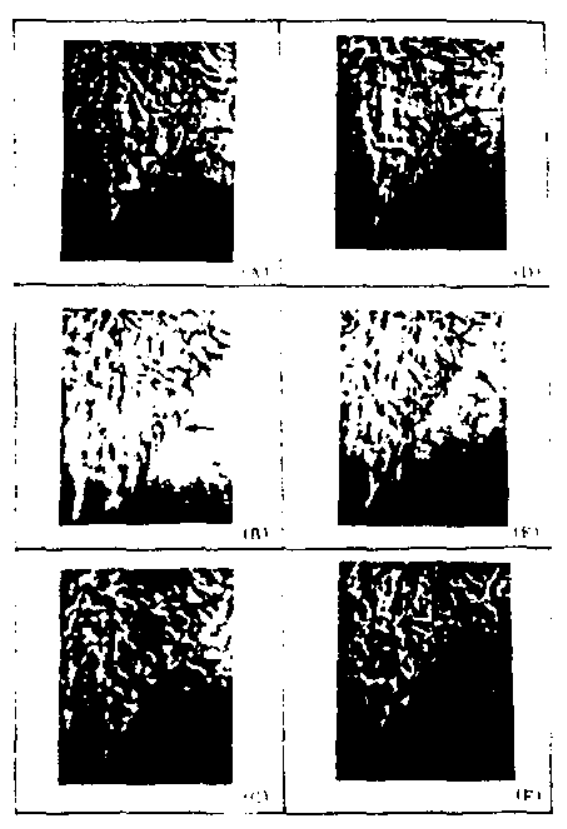

Figure 6, Schlieren Movie Showing Motton of an Individual Wrinkle

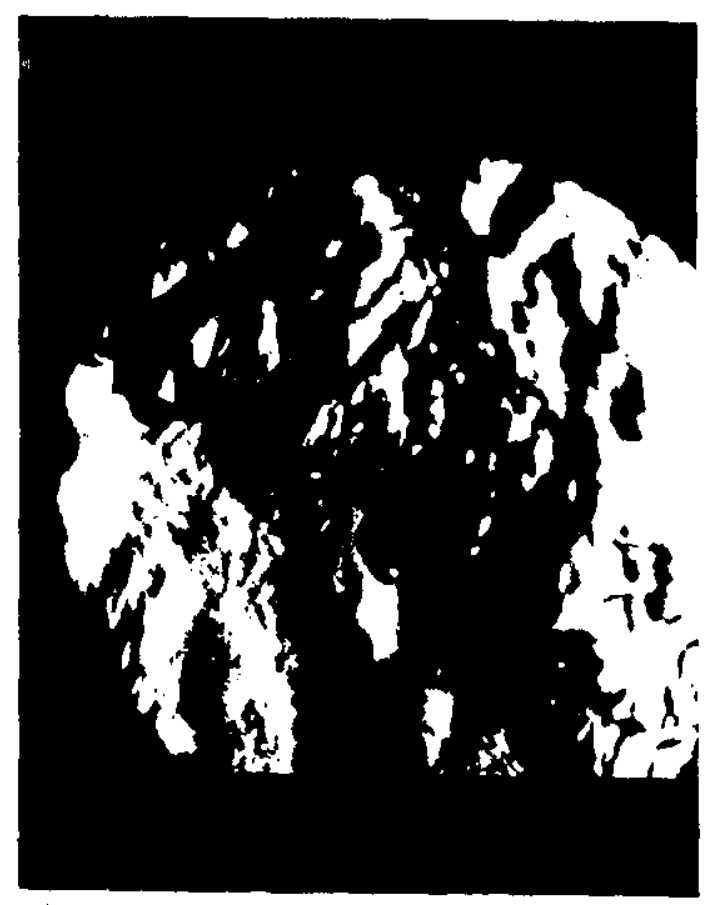

Figure 7. Structure of a Lean Propane-Atr Flame for $=0,89$ 


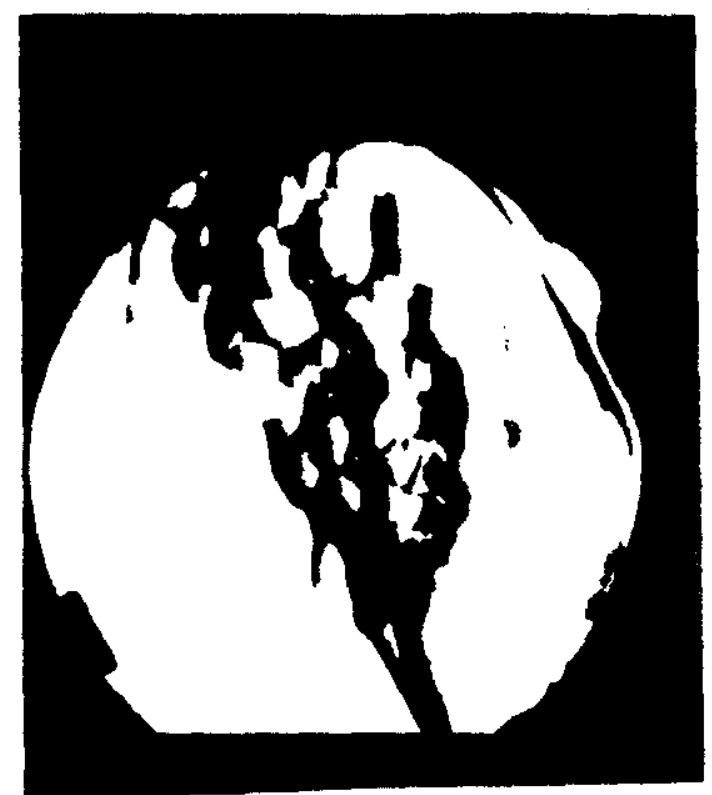

Figure 8. Low Reynolds Number Methane-Air Flame, $\mathrm{Re}_{\mathrm{M}}=17, \mathrm{~S}_{\mathrm{T}} / \mathrm{S}_{\mathrm{L}}=3.54$

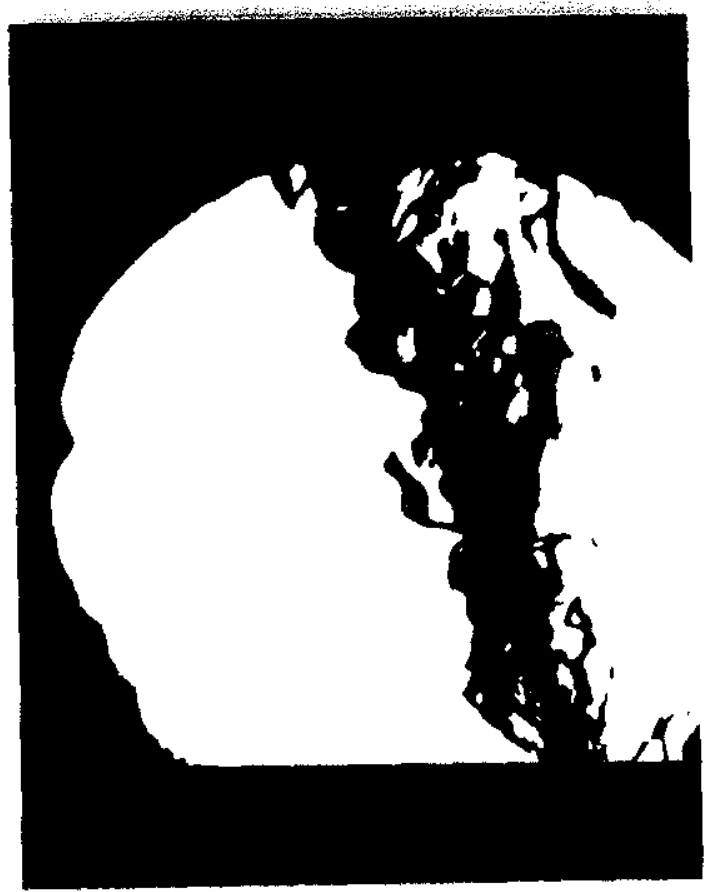

Figuxe 9. Intermediate Reynolds Number Flame $\operatorname{Re}_{M}=108, S_{T} / S_{L}=4.2$

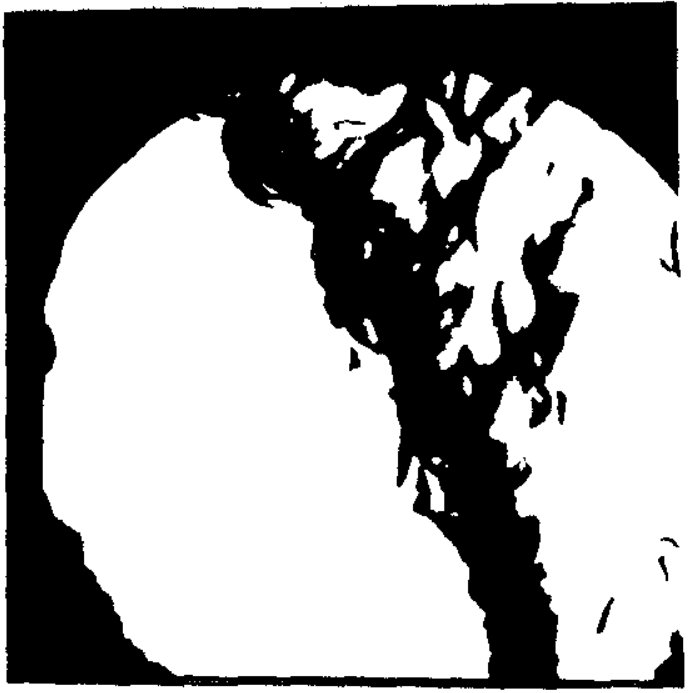

Figure 10. Higher Reynolds Number Methane-Air Flame, $R_{M}=450, S_{T} / S_{L}=4.9$

u.

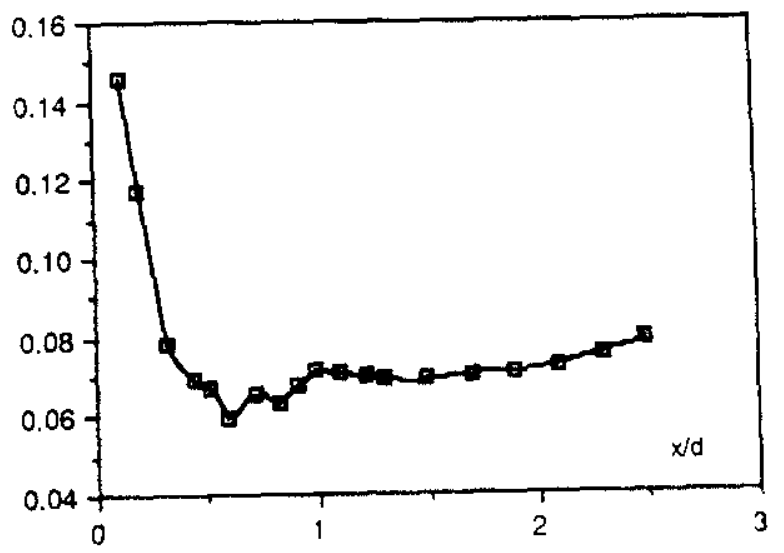

Figure 11. Decay of Turbulence Behind the Grid

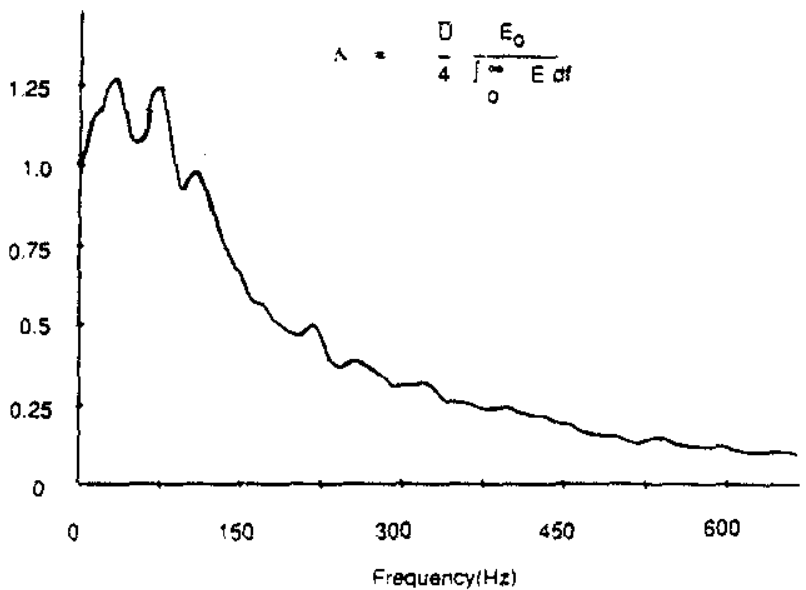

Figure 12。 Typical thot Wire Velocity Power

Spectrum in the Cold Flow at $x / d=0,9$ Integral $\mathrm{Scale}=1.8 \mathrm{ma}$. 